\title{
Automatic Scoring of Sister Chromatid Exchanges by Image Analysis in a Dose Response Experimenf
}

\author{
J.M. García-Sagredo, J. Piper, D. Rutovitz, J.J. Vaquero, and Yolanda Vázquez-Mazariego \\ Department of Medical Genetics, Hospital Ramón y Cajal (J.M.G.-S., Y.V.-M.), Department of \\ Bioengineering, ETSI Telecomunicación, Universidad Politécnica de Madrid (J.J.V.), Madrid, Spain; MRC \\ Human Genetics Unit, Western General Hospital, Edinburgh, UK (J.P., D.R.)
}

\begin{abstract}
A system which automatically selects second division metaphases and then, automatically scores the number of SCEs of each cell is described. In an initial set of experiments, the performance of the components of the system was measured using a data set in which metaphases had been visually classified as either 2 nd division or other; and in 2nd division metaphases, every SCE had been marked on a hard copy. SCE scoring had a true positive rate of about $75 \%$ and a false positive rate of about 1.5 false SCEs per metaphase analyzed. Second division
\end{abstract}

detection had a true positive rate of $80 \%$ and a false positive rate of about $10 \%$ of the non-2nd division cells. Next, the overall system was compared to human visual scoring in a dose-response experiment by analyzing the effect of mitomycin C on human chromosomes scored visually by two observers and by the fully automatic scoring. Human visual scoring and machine analysis showed similar dose responses, but the variability between them was considerable.

Key words: SCE, automatic scoring of SCEs, automatic selection of $2 \mathrm{n}$ division cells, image analysis.

\section{INTRODUCTION}

A sister chromatid exchange (SCE) is an interchange between homologous loci of DNA on sister chromatids. Many external factors have been shown to increase the frequency of SCEs. Since Wolff and Perry [1974] first described the FPG staining technique in which SCEs can be visualized by exposing dividing cells through two cell cycles to 5-bromodeoxyuridine (BrDU), leading to a differential or "harlequin" staining of sister chromatids, the SCE test has become a commonly used method for estimating genotoxic exposure. Major applications of the test include the use of lymphocyte SCE frequencies to estimate genotoxic exposure to human populations, and the use of human lymphocytes in vitro to investigate potential genotoxic agents.

The test requires counting the number of SCEs and the number of chromosomes in each metaphase scored. Due to individual response variations, and for consistency purposes of experimental design it is necessary to analyse a minimum of 25 metaphases per sample. Not all metaphases are suitable for SCE anaiysis. While oniy the $2 a d$ division cells show the required overall differential staining, there are usually also many 1 st and 3rd (or higher) division metaphases present, which implies that a considerably larger number of metaphases must be screened in order to identify the required number of 2 nd divisions. This is a tedious and time consuming task [García-Sagredo, 1990], and automation is clearly desirable [Lloyd, 1989].
Most research work in cytogenetics automation has concentrated on the problems posed by automatic metaphase finding and karyotyping [see the bibliography in Lundsteen and Piper, 1989], and almost all commercial development has restricted itself to this area. Partial automation of the SCE test is possible with the facilities offered by those commercial systems for cytogenetic analysis which provide automatic metaphase finding. This gives the possibility of visually selecting the 2 nd metaphases and analyzing them either on the screen or through the microscope, with computer accumulation of the results and computation of final frequencies [Shafer et al., 1986; García-Sagredo, 1990]. This can increase the speed of the analysis by a significant factor, besides making it more convenient.

Since the visual analysis of SCEs is straightforward and quite rapid (compared, in particular, with constitutional karyotyping), computer analysis will only be cost-effective if all stages are fully automated and operator involvement is negligible [Piper, 1990]. Available karyotyping systems rely on substantial operator intervention at all stages; during image acquisition, for assistance with segmenting chromosome clusters, and for correction of the final positions of the

Address reprint requests to Dr. I.M. García-Sagredo, Department of́ Medical Genetics, University Hospital Ramón y Cajal, 28034-Madrid, Spain. 
chromosomes in the karyogram. In contrast, a complete system for SCE analysis requires fully automatic metaphase search, digitisation at high resolution, segmentation of individual chromosomes, as well as selection of 2 nd division metaphases, analysis of SCEs, and accumulation of results. In respect of this requirement for a high level of automation and correspondingly minimal operator interaction, the task is similar to other systems that score many metaphase cells, notably those for analysis of dicentric chromosomes for radiation dosimetry [Bayley et al., 1981].

The feasibility of automatic scoring of SCEs has been demonstrated by several authors [Zack et al., 1976, 1977; Shafer et al., 1980; He and Chai, 1990], but not so far in the context of a complete system applied to routinely prepared samples. Except that Zack et al. [1977] tackled the problem of identifying correctly segmented chromosomes automatically, previous work in this field lacked three essential capabilities: automatic detection of 2 nd divisions, automatic chromosome segmentation, and discrimination between centromere and chromosome arm exchanges [Lloyd, 1989]. Here we describe and measure the performance of a pilot system which, while not yet fully integrated on a single automated microscope and computer, contains all necessary components for a complete system.

The ultimate aim of SCE analysis is to distinguish a raised SCE frequency from a background or control level, and to compare different frequencies, for example, when establishing a dose response. Conventional visual scoring obviously has several sources of variability, and the final acceptance of a machine system will depend on whether or not it leads to unacceptably greater experimental variability. To investigate the machine versus human dose-response, we measured with different observers the effect of Mitomycin C (MMC) on SCE frequency in human chromosomes in vitro. MMC was used because of its known mutagenic capacity, especially as an SCE inducer; it is a crosslinking agent, which acts as a bifunctional alkylating agent capable of inducing intrastrand and interstrand DNA crosslinks [Latt et al., 1975; Fujiwara et al., 1977; Tomasz et al., 1987].

\section{MATERIALS AND METHODS}

\section{Lymphocyte Culture}

Blood from a single healthy donor was cultured in vitro for $72 \mathrm{hr}$. The culture medium was RPMI supplemented with $20 \%$ fetal calf serum. BrDU was added at a concentration of $10 \mu \mathrm{g} / \mathrm{ml}$ for the last $48 \mathrm{hr}$ of culture. After harvesting the cultures, the slides were stained for $20 \mathrm{~min}$ in a $0.5 \mu \mathrm{g} / \mathrm{ml}$ solution of the fluorochrome Hoechst 33258 ; and after exposure to UV light for $12 \mathrm{hr}$ were incubated at $2 \times \mathrm{SSC}$ at $60^{\circ} \mathrm{C}$ for $2 \mathrm{hr}$.

\section{Image Digitization and Pre-Processing}

Following automatic metaphase finding, digitised metaphase images were obtained on a Magiscan-2 (Applied Imaging International, Warrington, $U K$ ) via a $\times 100$ objective and video camera. The image size was $512 \times 512$, 6-bit pixels. Images were transferred to a Sparcstation-1 (Sun Microsystems, Mountain View, CA). Each pixel value in the 6-bit image was multiplied by 4 and subtracted from 255 . This resulted in 8 -bit images with a grey scaie in which darker pixels were represented by higher values. For some of the initial experiments, additional cells were digitized fully automatically on the MRC Human Genetic Unit's FIP, which also produces 8-bit images with a grey scale in which darker pixels have higher values [Stark et al., 1989; Bayley et al., 1991]; subsequent image processing and analysis was identical to that described for images digitized by Magiscan- 2 .

Individual chromosomes were segmented from the metaphase images by automatically-chosen threshold followed by a fully automatic segmentation program. This used image shape and grey level analysis to recognize and correctly segment the majority of clusters of touching and overlapping chromosomes in a metaphase, and also recognised and rejected interphase nuclei and other non-chromosomal material [Ji, 1989a,b, 1994]. A substantial proportion (often $20 \%$ or more) of chromosomes were involved in clusters after the initial segmentation by threshold, and so fully automatic recognition and segmentation of the clusters increased the number of chromosomes available for analysis. Sometimes the segmentation program made a wrong decision, resulting in segmented objects that were not in fact single whole chromosomes. Because our system functioned fully automatically without operator intervention, such objects were analyzed as if they were single chromosomes, and in some cases this resulted in either false negative or false positive SCE events. Some metaphases were rejected at the segmentation stage as "impossible to segment."

The contrast of the set of segmented chromosomes was enhanced and standardized by transforming the pixel density histogram by histogram equalization followed by an exponential transformation of pixel values. This resulted in a reproducible, large difference in the values of central pixels in the light and dark chromatids.

\section{Automatic Detection of SCEs}

In earlier reported work, there have been two distinct approaches to locating SCEs on chromosomes. Zack et al. [1977] and Shafer et al. [1980, 1986] located potential SCEs where separate darkly stained regions found by thresholding are closely adjacent. This method tends to find a false SCE at the centromere, whether there is in fact an exchange or not. Zack et al. [1976] and He and Chai [1990] instead analyzed the ratio of the staining intensity along the two chromatids delineated by the chromosome medial axis, locating crossover positions of the darker staining intensity. With this method, only if there is an exchange at the centromere will a false SCE be detected. Here we also find the ratio of staining intensity along the individual chromatids; we improve on previous methods by locating the centromere explicitly, so that centromere exchanges may be rejected (or counted separately if so desired).

Chromosome axes, "shape" profiles, and centromeres were found by the methods described by Piper and Granum [1989]. Additional "half-profiles" of integrated density on either side of the axis were computed. The halfprofiles were smoothed to reduce high frequency noise. SCEs were detected where the two half-profiles for a single chromosome crossed (Fig. 1). A proportion of these "crossover" positions were not in fact at true SCEs, and the number of false positive SCEs was reduced by the following strategies:

1. The crossover was required to satisfy a quantization condition, that on either side of the crossover (moving along the chromosome axis) the difference between the two profiles exceeded some proportion of the common peak value.

2. Many false positive crossovers occurred at chromosome tips, on account of the chromatids separating, with one appearing slightly longer than the other. In order to reduce these, the crossover was rejected if it was too close to the end of the "storter" chromatid, or if the density level at which the haif-profiles cross was less than some proportion of the common peak value.

If the crossover was located within four pixels (about $0.5 \mathrm{~mm}$ ) of the machine-found centromere, then it was classified as a "centromere exchange" (CE) rather than an $\mathrm{SCE}$, and discarcied. 

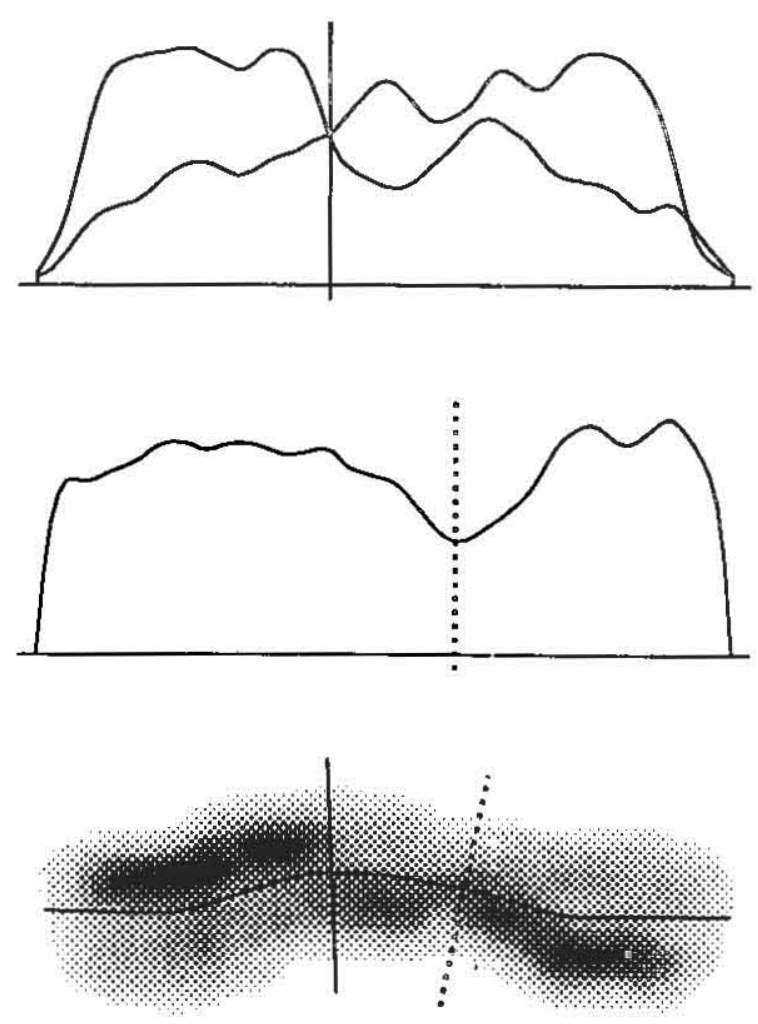

Fig. 1. A chromosome showing the computed medial axis. Above it is the "shape" profile, from which the centromere (broken line) is computed. At top are the two density half-profiles; the SCE (full line) is found where these cross. Note the insignificant crossing that has been ignored at the short arm telomere.

\section{Determination of SCE Classification Rates}

Hard copy images of about 80 digitized, analyzable second divisions were obtained. Following visual analysis on the display screen (and using the microscope in cases of doubt), every SCE was marked in ink on the hard copy in order to provide the "truth standard." The SCE classifier parameters (quantization, end conditions, etc., as described above) were selected to give satisfactory performance on about half of the data; the remainder was then used as an independent test set. The true positive frequency was measured as the proportion of the marked SCEs that were found by machine. In addition, the variability among cells was measured by the correlation coefficient $r$ between the visual and automatic scores for each cell.

\section{Automatic Classification of 2nd Division Metophoses}

In first divisions every chromosome is homogeneously and darkly stained, while in second divisions every chromosome is hariequin stained; these can easily be discriminated. In thirs divisions, some cinromosomes (or parts of some chromosones) will be harlequin stained, while the remainder will be uniformly pa'e. Occasionaily, only a very small part of the metaphase will not show the hariequin staining. Sach ceils are difficult to distinguisi from second divisions, but should be relatively rare. Eloyd [1989] reported that the proportion of 2 nd divisions in a cell population found by a metaphase finder could be increased from $20 \%$ to between $60 \%$ and $70 \%$ by judicious choice of metaphase finder parameters. For a fully automatic analysis system, this level of performance would not be ade- quate. Here, automatic discrimination of 2nd divisions was based on analysis of the full resolution metaphase image. Two whole-cell features were computed from the set of half-profiles of the segmented chromosomes:

1. A "ratio-profile" was computed for each chromosome, being at each point the ratio of the absolute difference to the sum of the halfprofiles. For harlequin stained regions, this ratio should be large, while for uniform staining it should be close to zero. The mean ratio-profile value for the cell was computed, and discriminated first divisions, and those third divisions with only a small proportion of harlequin staining (both having a small value of the feature) from second divisions (large value).

2. The set of per-chromosome mean values of the ratio-profile was computed. The coefficient of variation (c.v. = standard deviation/ mean) of the set discriminated first and second divisions (small c.v.) from most third divisions (larger c.v.). The computation was appropriately weighted by chromosome length.

In either case, regions of the chromosome profiles immediately adjacent either to the tips of the arms, or to the centromere, were excluded, since in both of these locations the chromatid structure may be atypical, resulting in atypical profile values.

A simple classifier was constructed by setting a threshold for each of the two features independently; these were chosen from a plot of the feature distributions for a training set of metaphases digitized without human selection, and the classifier was then applied to an independent test set. The truth standard was marked on hard copies of the digitized images; in cases of doubt (some $3 \mathrm{rd}$ divisions are hard to distinguish from 2nd division) cells were relocated on the microscope and the decision taken via the eyepieces.

\section{Dose-Response Experiment}

Whole blood was taken from 3 healthy donors, and cultivated in vitro for $72 \mathrm{hr}$ as described above, while being exposed during the whole culture time to different concentrations of MMC (Kiowa) diluted in distilled water. The cultures were carried out in batches of 5 cultures per donor, with MMC concentrations of 0 (control), 2, 8, 12, and $16 \mathrm{ng} / \mathrm{ml}$.

Each slide was scored blind. Since the automatic system will frequently make segmentation errors, it is unrealistic to restrict scoring to those cells containing exactly 46 chromosomes. Instead, all metaphases that apparently had a normal chromosome complement were analyzed. Simultaneously with the SCE score the chromosome number was counted, the apparent range lying between 35 and 47 chromosomes per cell. SCE frequencies were therefore calculated both as SCEs/cell (taking no account of the apparent number of chromosomes) and SCEs/chromosome.

In order to provide a basis for relating the differences between machine and human scoring, each slide was analyzed visually in three different ways and by two observers as follows:

a. Observer 1 found a set $S_{1}$ of cells manualiy and analyzed them through the microscope.

b. Observer 2 analyzed visually through the microscope a set $S_{2}$ of cells found by the Magiscan metaphase finder.

c. Observer 1 visually analyzed the same set $S_{2}$ of cells from digital images presented on the Magiscan screen.

The computer system aiso analyzed $\mathrm{S}_{2}$; a few cells were rejected by the segmentation program (see above). The resulting frequencies were compared by one-way analysis of variance; the frequencies observed in $S_{2}$ were compared pair-wise by the correlation coefficient $r$. 
TABLE I. SCE Detection Rates

\begin{tabular}{lccccc}
\hline & $\begin{array}{c}\text { No. cells } \\
\text { scored }\end{array}$ & SCEs & $\begin{array}{c}\text { True positive } \\
\text { SCEs found (\%) }\end{array}$ & $r$ & $\begin{array}{c}\text { False positive } \\
\text { SCEs (per cell) }\end{array}$ \\
\hline Training set & 50 & 354 & $266(75 \%)$ & 0.77 & $64(1.3)$ \\
Test set & 34 & 245 & $184(75 \%)$ & 0.72 & $52(1.5)$ \\
\hline
\end{tabular}

TABLE II. 2nd Division Detection Rates

\begin{tabular}{lcccr}
\hline & & & \multicolumn{2}{c}{ Machine classification } \\
\cline { 3 - 5 } & True classification & No. of cells & 2nd-div & Other \\
\hline \multirow{2}{*}{ Training set } & 2nd-div & 31 & 29 & 2 \\
\multirow{3}{*}{ Test set } & other & 60 & 7 & 53 \\
& 2nd-div & 119 & 99 & 20 \\
& other & 166 & 12 & 154 \\
\hline
\end{tabular}

TABLE III. SCEs/Cell Average

\begin{tabular}{|c|c|c|c|c|c|c|}
\hline \multirow[b]{2}{*}{ Observer } & \multirow{2}{*}{$\begin{array}{c}\text { Cell } \\
\text { set }\end{array}$} & \multicolumn{5}{|c|}{ MMC dose $(\mathrm{ng} / \mathrm{ml})$} \\
\hline & & Control & 2 & 8 & 12 & 16 \\
\hline 1 & $S_{1}$ & 6.1 & 7.0 & 10.3 & 11.1 & 11.9 \\
\hline 2 & $\mathrm{~S}_{2}$ & 5.1 & 7.3 & 11.1 & 9.5 & 13.5 \\
\hline 1 & $\mathrm{~S}_{2}$ & 5.2 & 7.8 & 11.0 & 9.9 & 13.6 \\
\hline Machine & $\mathrm{S}_{2}$ & 6.1 & 8.4 & 9.7 & 9.9 & 10.9 \\
\hline
\end{tabular}

\section{RESULTS}

Table I shows SCE detection performance on both the training and test sets, and Table II shows the performance of the 2nd division classification.

Table III and Figure 2 illustrate the average SCEs/cell found by the 3 sets of visual observations and by the automatic system for the control cultures and the 4 levels of MMC exposure. Figure 3a shows cell-by-cell comparison scores between the two observers $(r=0.92)$; Figure 3b shows the cell-by-cell comparison between the machine scores and the mean score of the two observers $(r=0.72)$.

One-way analysis of variance of the data in Table III indicated that while the differences between doses were significant $(P<0.001)$, only at the highest MMC dose were the differences between observers significant $(P<0.005)$.

The data presented are in units SCEs/cell. Corresponding results with data expressed as SCEs/chromosome are not presented but were essentially similar.

\section{DISCUSSION AND CONCLUSIONS}

Overall, about three-quarters of all SCEs were correctly detected, with a false positive rate of about 1.5 per cell. The correlation between visual and automatic scoring on a cell by cell basis was greater than 0.7 in all experiments. We expect that the performance would improve if a linear or quadratic discriminant classifier were substituted for the simple box classifier used in the experiments reported here.
This would permit inclusion of a further valuable feature, the relative slopes of the half-profiles at the crossover.

SCEs were missed in particular where the rule rejecting crossovers close to the chromosome tip did in fact reject a true SCE; others were lost if the machine-found axis or centromere were incorrect, or where a chromosome cluster went undetected or unsplit. False positives resulted from a variety of causes: twisting of chromosomes resulting in crossed chromatids; unsegmented or incorrectly segmented chromosome clusters; and in cells in which the dark and pale staining intensities were not very uniform.

The results show that the false positive rate for 2 nd division detection is only about one in ten of the true non-2nd divisions, while more than $80 \%$ of true 2 nd divisions are recognized correctly. Considering that an approach based on the average properties of the staining will not detect those third divisions that have only a very small proportion of non-harlequin stained material, the results are encouraging, and it is likely that this level of performance would prove adequate in a fully integrated system. In order to make further improvement, it will be necessary to analyze individual chromosomes, and determine if they are non-harlequin. The problem is that there will always be a proportion of objects in the cell that pass such a criterion when measured automatically, simply because the underlying processes (automatic segmentation, axis fitting, etc.) have a small failure rate. What is required is a way of distinguishing those chromosomes for which the automarically-obtained measure- 


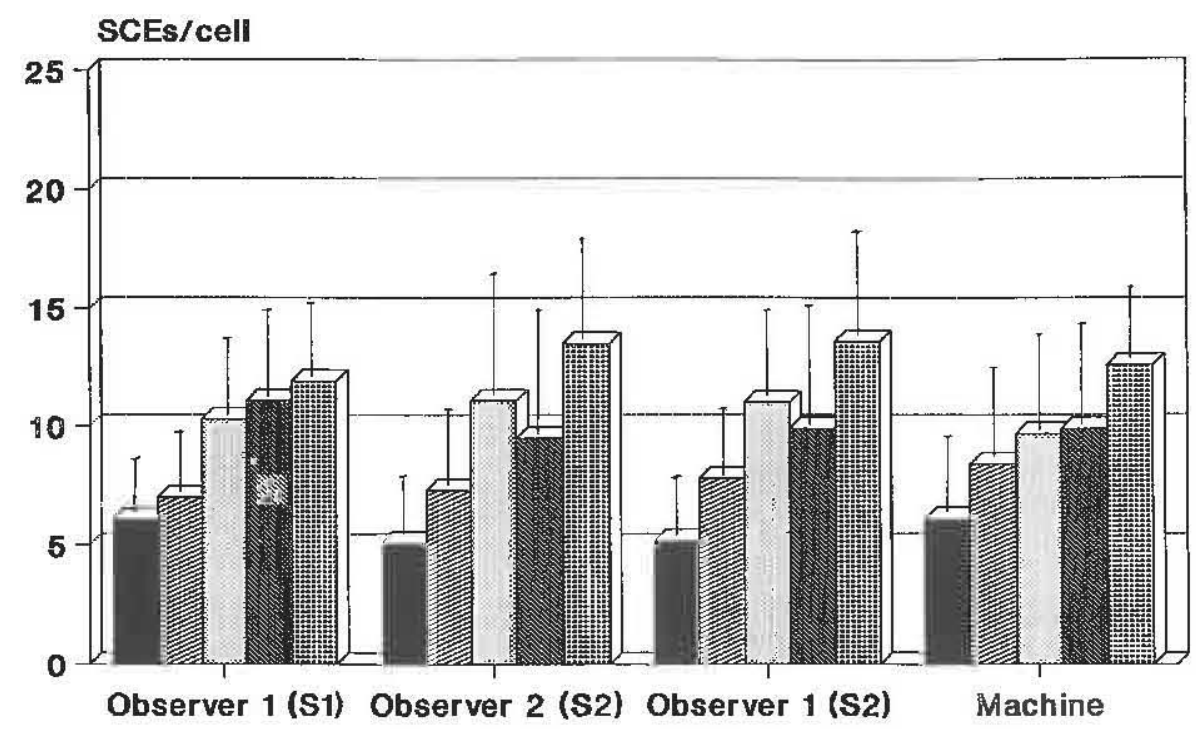

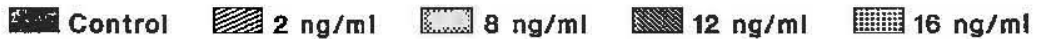

Fig. 2. The mean scores obtained by four sets of observations and five MMC doses.

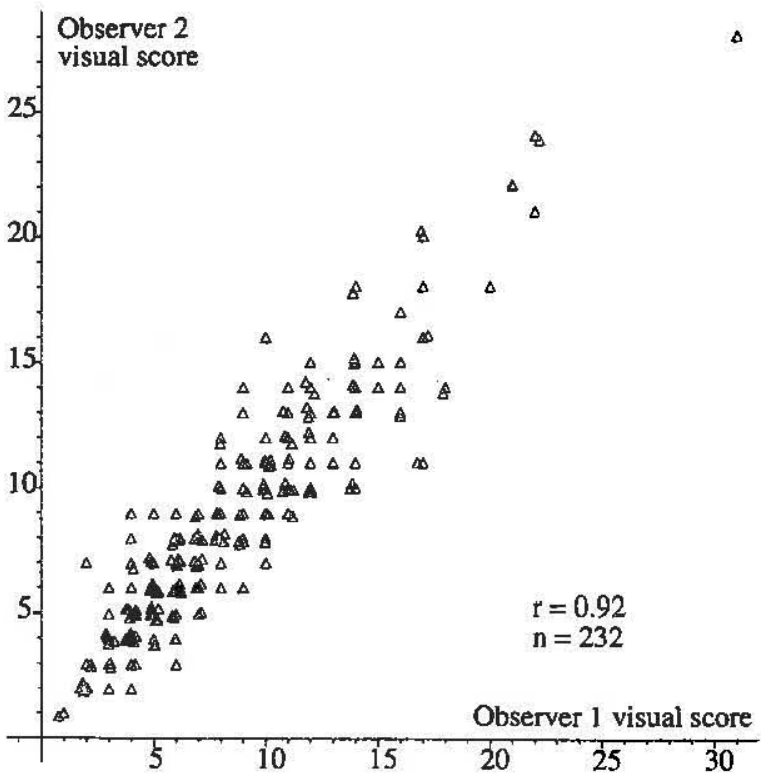

(a)

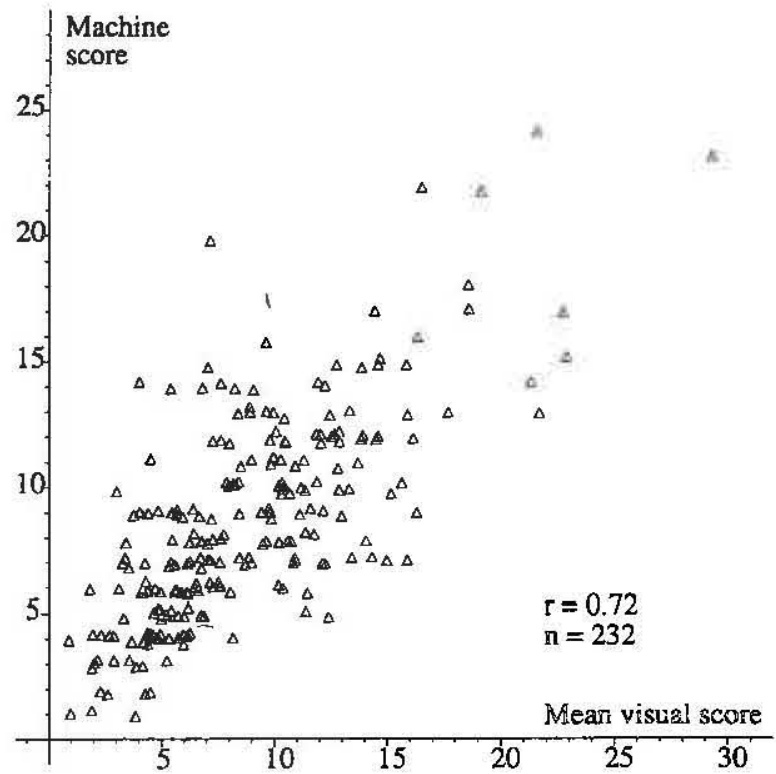

(b)

Fig. 3. a: Individual cell scores by two observers on the same set of 232 cells. The data positions have been modified by small random perturbations in order to show the frequency of the more common points. $\mathbf{b}$ : $A$ similar comparison on the same set between machine scores and the mean of the two visual ooserver scores.

ments are reliable, and basing the results only on such chromosomes. Such a "guaranteed high quality chromosome" detector would be generally useful in many applications in automated cytogenetics.

A system which relies on automatic metaphase selection and automatic chromosome segmentation will result in a set of metaphases with a wide range in the apparent chromosome number, with an apparently low number (i.e., <46) being more frequent than an apparently high number on account of chromosomes involved in unrecognised and therefore unsegmented clusters. For this reason, we accumulated data in the units SCE/chromosome (data not pre- 
sented) as well as SCE/cell. There was no noticeable difference between the two sets of results (data not presented). In practice, with rare exceptions, our material lay in the range 43 to 47 chromosomes/cell.

In the dose response experiment, both manual and automatic scoring showed, as expected, a trend for an increased rate of SCEs from the controls to the higher MMC concentrations.

The mean scores of observers 1 and 2 agree closely when analysing the same set $S_{2}$ of cells even though one was using a microscope and the other the Magiscan digitised images. However, Figure 3a shows that in fact there is quite a wide divergence between the two observers when scores on individual cells are compared. When observers 1 and 2 scored different sets of cells $\left(S_{1}\right.$ and $\left.S_{2}\right)$ from the same set of slides, even the mean scores differed noticeably, especially on cultures with the two highest concentrations of MMC. We note that although $S_{1}$ and $S_{2}$ were selected differently, they are drawn from the same slides and may therefore be expected to have a substantial (but unknown) proportion of metaphases in common.

The mean scores from the machine showed a similar increasing rate of SCEs as dose increased. It is notable that only the machine scores show the expected monotonic relationship with MMC dose, but this observation should be treated with caution given the wide discrepancy between machine and visual scores on individual cells (Fig. 3b).

The results obtained demonstrate that fully automatic analysis of SCEs with adequate accuracy is feasible. In an integrated system with computing resource equal, say, to a Sparcstation-1, the throughput would be better than 5 cells analyzed per minute; we expect that such a performance would be quite acceptable.

\section{ACKNOWLEDGMENTS}

We thank Liang Ji for advice, and Jill Gordon and Margaret Stark for assistance with metaphase finding and digitisation. This study was supported by the European Community's Concerted Action on Automated Cytogenetics (EC Medical and Health Research Program, project no. II.1.1/ 13).

\section{REFERENCES}

Bayley R, Carothers A, Chen X, Farrow S, Gordon J, Ji L, Piper J, Rutovitz D, Stark M, Wald N (1991): Radiation dosimetry by automatic image analysis of dicentric chromosomes. Mutat Res 253:223-235.

Fujiwara Y, Tatsumi M, Sasaki MS (!977): Cross-link repair in human cells and its possible defect in Fanconi anemia cells. J Mol Biol 113:635-649.

García-Sagredo JM (1990): Performance of a semiautomatic system for chromosome analysis in scoring aberrations: Appüication to analyze the effect of low-level $50 \mathrm{~Hz}$ electromagnetic fields on human chromosomes in vitro. In Burger G, Oberholzer M, Peter Voijs G (eds): "Advances in Analytical Cellular Pathology." Amsterdam: Excerpta Medica, pp 65-66.

He L, Chai Z (1990): A new method of automatic SCE detection. Pattern Recogn 23:95-97.

Ji L (1989a): Intelligent splitting in the chromosome domain. Pattern Recogn 22:519-532.

Ji L (1989b): Decomposition of overlapping chromosomes. In Lundsteen C, Piper J (eds): "Automation of Cytogenetics." Heidelberg: Springer-Verlag, pp 177-190.

Ji L (1994): Fully automatic chromosome segmentation. Cytometry (in press).

Latt SA, Stetten G, Juergens LA, Buchanan GR, Gerald PS (1975): Induction by alkylating agents of sister chromatid exchanges and chromatid breaks in Fanconi's anemia. Proc Natl Acad Sci USA 72:40664070.

Lloyd DC (1989): Automated aberration scoring: the requirements of an end-user. In Lundsteen C, Piper J (eds): “Automation of Cytogenetics." Heidelberg: Springer-Verlag, pp 9-17.

Lundsteen C, Piper J (eds) (1989): “Automation of Cytogenetics." Heidelberg: Springer-Verlag.

Piper J, Granum E (1989): On fully automatic feature measurement for banded chromosome classification. Cytometry 10:242-255.

Piper J (1990): Automated cytogenetics in the study of mutagenesis and cancer. In Obe G (ed): "Advances in Mutagenesis Research 2." Berlin: Springer-Verlag, pp 127-153.

Shafer DA, Swenson R, Oney D, Falek A, Maden JJ (1980): Computer image analysis of sister chromatid exchanges. Am J Hum Genet 32:88A.

Shafer DA, Mandelberg Ki, Falék A (1986): Computer automation oĩ metaphase finding, sister chromatid exchange, and chromosome damage analysis. In de Serres FJ (ed): "Chemical Mutagens: Principles and Methods for their Detection," vol 10. New York: Plenum Press, pp 357-380.

Stark M, Farrow S, McKie M, Rutovitz D (1989): Automatic high resolution digitisation of metaphase cells for aberration scoring and karyotyping. In Lundsteen C, Piper J (eds): "Automation of Cytogenetics." Heidelberg: Springer-Verlag, pp 31-43.

Tomasz M, Lipman R, Chowdary D, Pawlak J, Verdine GL, Nakanishi K (1987): Isolation and structure of a covalent cross-link adduct between mitomycin C and DNA. Science 235:1204-1208.

Wolff S, Perry P (1974): Differential Giemsa staining of sister chromatids and the study of sister chromatid exchange without autoradiography. Chromosoma 48:341-353.

Zack GW, Spriet JA, Latt SA, Granlund GH, Young IT (1976): Automatic detection and localisation of sister chromatid exchanges. J Histochem Cytochem 24:168-177.

Zack GW, Rogers WE, Latt SA (1977): Automatic measurement of sister chromatid exchange frequency. J Histochem Cytochem 25:741753. 